\title{
Research on Urban Planning Management Problems Based on GIS
}

\author{
Gang Li \\ Management Committee of Airport New Zone of Shaanxi Province Xixian New District, Xi'an, Shaanxi, \\ 712034, China
}

Keywords: GIS, Urban planning management, Problems and application aspecs

\begin{abstract}
As a product of the combination of geographic information system (GIS) and urban planning industry, city planning management information system provides fast and effective information acquisition methods and management methods for city planning management, thus continuously improving the efficiency and level of planning management. Based on the in-depth study of the related technologies of urban planning management information system, this paper proposes the application plan of GIS technology in urban planning management information system for urban planning management of large and medium-sized cities. The urban planning management department has the right to approve land use for urban construction units and exercise the approval management function of "one book and two certificates" (construction project site selection opinions, construction land planning permits, construction project planning permits) in urban planning management, The daily business work of the planning and management department revolves around this approval management function. All the geospatial data and system management tables in this system are stored in the Oracle $10 \mathrm{~g}$ system, which realizes the storage in a full-relational manner, and the large-scale relational database management system technology can be used to more efficiently establish a geographic information spatial database.
\end{abstract}

\section{Introduction}

In urban planning management, it has become a consensus in the field of urban planning to use GIS as the core technology. In recent years, the application of GIS in my country's urban planning management industry has developed rapidly. The application of GIS technology provides fast and effective information acquisition methods and information analysis methods for urban planning and management, and provides new planning management techniques, new forms of planning scheme expressions, new forms of public participation and public supervision mechanisms, and new governments Strong support from the public image of the department has continuously improved the work efficiency and technical level of the city planning management business. Urban planning management is one of the important fields of GIS application. Using the data collection function of GIS can improve the efficiency of obtaining urban planning and construction information, and conveniently input multiple data sources and various types of urban planning and construction information into the database system; using the information query function of GIS can quickly provide Various urban planning and construction information (including spatial information, attribute information, statistical information, etc.) required by users; using GIS database management functions, can automatically manage massive urban planning and construction data (including basic geographic data, red line data, completion data Etc.), and work on the creation, operation, and maintenance of urban planning and construction databases; using the statistical mapping function of GIS, a large number of abstract urban planning and construction data can be displayed in a visual form (such as thematic maps or statistical maps) to display various cities Planning thematic content; using the professional model application function of GIS, can carry out urban planning and construction prediction, evaluation, planning, simulation and decision-making; using the output function of GIS, it can support multimedia presentation and output of urban planning and construction information given to multiple media. In short, GIS is indeed an effective tool for efficient analysis, utilization and management of urban planning and construction information with obvious spatial characteristics. The acquisition and processing of urban 
construction information is suitable and one of the areas in which GIS is most in need.

\section{Features and Advantages of GIS-Based Urban Design Work Methods}

Urban design work begins with data collection and status analysis. At present, it is inefficient to directly use the project database to summarize electronic data. Because the current status information is scattered in multiple independent drawings and documents, it is difficult for the designer to form a complete understanding of the base. One of the advantages of GIS is the efficient organization and management of massive information from multiple sources. The urban design work method based on GIS has established the geographic information database of the base from the beginning of the project based on the ArcGIS platform, and input multi-source data in the form of layered layers, so as to summarize all the information of the base in the same one based on spatial geographic coordinates On the platform, and realize two-way query and association. After the database is built, the designer can directly use ArcGIS spatial analysis methods to carry out thematic analysis of the base according to the characteristics and design goals of the base, such as terrain analysis, status quo construction, and facility service area evaluation. On the basis of each special analysis, ArcGIS overlay analysis technology can be used to derive new data layers on the basis of overlaying two or more data layers, so as to explore the relationship between different information and deepen the understanding of the base. According to the important parameter variables that affect the design, the above-mentioned means can be used to establish a parameter model to conduct a comprehensive evaluation of the site's land suitability and land use value, etc., to provide a basis for refined scheme design). In recent years, with the rapid development of GIS 3D analysis technology, base 3D scene models can be quickly created in ArcGIS, allowing designers to intuitively feel the topography and site atmosphere, and can perform 3D quantitative analysis such as landscape sight, engineering earthwork, etc. Therefore, the pre-analysis of urban design is extended from traditional two-dimensional to three-dimensional, and the ability to grasp the space is strengthened.

\section{Application of GIS in Urban Planning}

Urban planning refers to the rational determination of urban development goals, urban nature, scale, and layout in accordance with the country's urban development and construction guidelines and the city's natural and construction conditions, with a strong emphasis on land use space allocation and urban industry and infrastructure in the planned area Layout. The core of urban planning is the planning of urban material space. It must provide physical space for non-material planning objects such as urban economic industry, policy measures, and space is the protagonist of urban planning. In addition to the massive data processing and management functions, GIS also has a powerful spatial analysis function. The core of urban planning is to scientifically and rationally make urban physical space planning decisions. The two have mutual reference and absorption in "space" The point of fit.

The application of the spatial analysis function of GIS in the preparation of urban planning mainly includes urban spatial expansion and urban landscape pattern analysis research, urban construction land suitability evaluation, urban public facilities site selection research, urban transportation network research and so on. The most commonly used traditional GIS spatial analysis modules in these studies are spatial information query and measurement, buffer analysis, overlay analysis, network analysis, etc. In recent years, with the advancement of GIS technology, the combination of various planning models in urban planning and GIS can complete complex spatial decision-making problems. These models include site selection model, location-configuration model, cellular automaton model, and urban construction. Multi-criteria decision-making model for land suitability evaluation. The traditional site selection model is based on the city's digital elevation model (DEM), using GIS buffers and overlay analysis to generate site selection thematic maps for city planning and decision-making services. The location-allocation (LA) model is used to determine the optimal location of various public service facilities and infrastructure in the city, 
thereby optimizing the spatial arrangement of certain facilities and resources. The combination of LA model and GIS is a powerful tool for solving such complex spatial decision problems. It provides an intuitive and accurate basis through GIS data processing and calling, model calculation, and GIS thematic map display. It can present the optimal location of resource allocation to the city Planning decision-makers, improve the scientific rationality of urban planning, and effectively avoid the randomness of subjective decision-making. The Cellular Automaton (CA) model is a model that can be used to simulate the urban spatial evolution process, and it has a strong spatial dynamic simulation capability. In recent years, the combination of CA and GIS has become closer and closer. The combination of the two has not only enhanced the spatial model calculation and analysis capabilities of GIS, but also made the application of CA technology in urban planning more in-depth. At present, the most widely used GIS technology in urban planning is the suitability evaluation model of urban construction land, which is the basic work of urban planning. Combining the multi-criteria decision-making (MCD) model with the basic functions of GIS can make GISbased construction land evaluation technology go deeper and better. The traditional land suitability evaluation only uses the superposition function of GIS, and does not reflect the influence of social, economic, environmental and other factors, and does not consider the relative importance and priority of these factors. Combining MCD technology and GIS, and using GIS as the evaluation technology platform can make land evaluation not only feasible in material form, but also meet various needs of economy, society and environment. With the development and application of 3D GIS technology, the theory and model of city planning are built on the 3D GIS platform, and the 3D GIS technology can be used to simulate the urban planning. This can not only predict changes in the state of urban planning, but also objectively evaluate and select various planning options, so that urban planning problems can be better solved. Practice has proved that applying the spatial analysis function of GIS to the preparation of urban planning can provide a scientific basis for determining the reasonable development scale and spatial layout of the city, which will inevitably improve the rationality and accuracy of the preparation of urban planning.

At present, GIS-based urban design methods still face many bottlenecks in practical applications. For urban designers who are used to traditional methods, the data structure and operation interface of GIS do not conform to their thinking and design habits. This article does not require urban designers to abandon traditional working methods and habits, but proposes new possibilities and prospects from the perspective of technological development. In recent years, although the main work platform for urban design is still AutoCAD, many urban design teams have begun to use the GIS platform and other new technologies for work support. It is foreseeable that urban design work will apply a variety of technologies and tools including GIS at the same time, and because of its unique advantages, GIS is very suitable for becoming a variety of technologies, multiple software platforms, multi-source information and even more This is an important platform for the integration of each work stage. Therefore, the author believes that the future participation of GIS in urban design will become higher and higher. Based on the GIS platform, it may become the main working method of urban design.

\section{Conclusion}

Since the "space” of GIS and urban planning are essentially the same, it is practically necessary and technically feasible to apply GIS technology to modern urban planning. The use of GIS technology in the urban planning system can provide strong support for the management and decision-making of urban planning. In the planning management, the massive database processing function of GIS can be used to construct the urban planning management information system to realize the modernization and automation of planning management. Using the spatial analysis function of GIS in planning, under the spatial modeling framework determined and provided by GIS, a variety of spatial decision models can be constructed for different purposes of urban planning, and auxiliary decision-making for complex spatial problems in planning, So that the urban planning scheme is more reasonable and feasible. Although the application of GIS in urban planning is becoming more and more extensive and deep, we should also see the limitations of GIS 
application. GIS is not a panacea, it cannot solve all problems in urban planning, and GIS will not work without the expertise of urban planning. At present, GIS only plays a role as a decision support tool in the planning management and preparation process. In the long run, the knowledge of urban planning experts and GIS can be organically combined to build a knowledge-based GIS system in order to give full play to GIS in urban planning. Application potential, thus achieving a closer integration of GIS and urban planning.

\section{References}

[1] Zeng Zhongping, Li Zonghua, Zhao Zhongyuan, et al. Research on Urban Planning Information System Based on 3D GIS. Civil Engineering and Environmental Engineering, vol. 29, no. 5, pp. 2630, 2007.

[2] Chen Yongmin, Chen Zhian. Development and Research of Urban Drainage Network Planning and Management System Based on GIS. Journal of Hunan University (Second Edition), no. 11, pp.127-131, 2002.

[3] Xiong Xuebin, Wang Yong, Guo Jiyuan. Design and Implementation of GIS-based Small and Medium City Planning Management Information System. Modern Computer (Professional Edition), vol. 12, no. 5, pp. 53-55, 2004.

[4] Zhang Heng, Li Gang, Feng Huili, et al. Research on the application of GIS-based urban planning information resource collaboration system. Planner, no. 7, pp. 80-83, 2011.

[5] Chen Lin. Research on GIS-based urban planning information management system. Commodities and Quality, vol. 11, no. 46, pp. 332-332, 2015. 